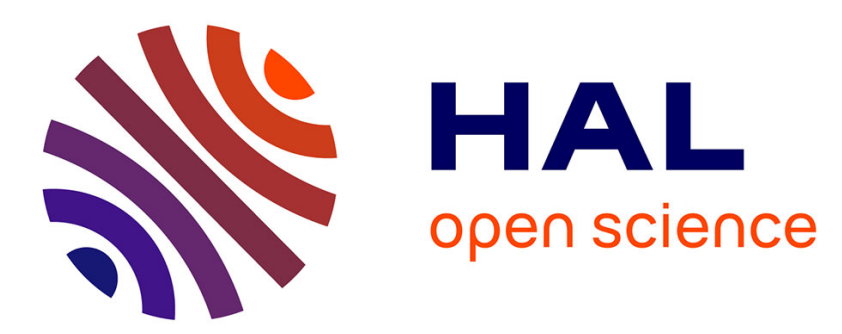

\title{
Calculs et optimisation des générateurs de courant hélicoïdaux par explosif
}

Etienne Auvray, Jacques E. Besançon, Alain Ducauze

\section{To cite this version:}

Etienne Auvray, Jacques E. Besançon, Alain Ducauze. Calculs et optimisation des générateurs de courant hélicoïdaux par explosif. Revue de Physique Appliquée, 1970, 5 (2), pp.301-308. 10.1051/rphysap:0197000502030100 . jpa-00243392

\section{HAL Id: jpa-00243392 https://hal.science/jpa-00243392}

Submitted on 1 Jan 1970

HAL is a multi-disciplinary open access archive for the deposit and dissemination of scientific research documents, whether they are published or not. The documents may come from teaching and research institutions in France or abroad, or from public or private research centers.
L'archive ouverte pluridisciplinaire HAL, est destinée au dépôt et à la diffusion de documents scientifiques de niveau recherche, publiés ou non, émanant des établissements d'enseignement et de recherche français ou étrangers, des laboratoires publics ou privés. 


\title{
CALCULS ET OPTIMISATION DES GÉNÉRATEURS DE COURANT HÉLICOÏDAUX PAR EXPLOSIF
}

\author{
Etienne AUVRAY, Jacques E. BESANÇON et Alain DUCAUZE \\ C. E. A., Centre d'Etudes de Limeil, B. P. 27, 94, Villeneuve-Saint-Georges
}

(Reçu le 17 novembre 1969)

\begin{abstract}
Résumé. - Nous proposons une étude théorique des générateurs de courant utilisant le principe du relèvement par explosif d'un cylindre placé sur l'axe et à l'intérieur d'une bobine solénoïde. Deux modèles différents sont proposés. Pour l'un le pas de la bobine est continûment variable; pour l'autre le pas présente plusieurs valeurs discontinues. Dans les deux cas, la variation du pas en fonction de la distance qui sépare une spire de la charge finale est calculée dans l'hypothèse d'un gain maximum de courant. Nous tenons compte des pertes principales dues à l'effet Joule résultant de la diffusion du champ à travers les spires. Les valeurs des paramètres ont été définies par les résultats expérimentaux afin que la théorie ainsi établie soit d'une utilité pratique. Enfin nous introduisons une perte supplémentaire d'énergie due à la rotation de la partie relevée du tube sous l'action des forces magnétiques. Ces dernières pertes sont négligeables pour les solénoïdes à grand gain bien qu'elles augmentent comme le carré des pertes par effet Joule.
\end{abstract}

\begin{abstract}
We study theoretically current generators using coil compression. Two designs have been considered. In one of them the pitch is continuously variable, in the other one a multistaged coil is used. In both cases the pitch variation versus the distance from an individual coil turn to the end-load is calculated with the condition that the current gain is maximum. The main losses due to Joule effect created by the field diffusion through the coil turns have been taken into account. The parameters have to be adjusted with experimental results in order that the theory is of practical use. We also consider another cause of energy losses, a side movement of the metal conductors due to the magnetic forces. The latter are negligible for high-gain coils, despite their increasing with the square power of the Joule effect losses.
\end{abstract}

I. Introduction. - De nombreuses expériences de physique exigent des sources d'énergie électrique susceptibles de fournir des impulsions de courant à la fois brèves et puissantes. Par exemple, on demandera des impulsions de courant supérieures au mégaampère délivrées en un temps de l'ordre de la microseconde, ce que ne peut réaliser une batterie de condensateurs conventionnels.

Les recherches s'orientent dans différentes directions : stockage d'énergie magnétique dans un supraconducteur, récupération de l'énergie cinétique d'une machine tournante ou de l'énergie chimique d'un explosif. Le problème le plus difficile est évidemment le transfert de ces énergies dans une charge généralement inductive.

L'objet de cette étude vise à la transformation de l'énergie chimique contenue dans un explosif en énergie électrique. L'explosif agissant par la pression des gaz brûlés, provoque la déformation d'un circuit dont l'inductance décroît très rapidement. Cette décroissance entraîne corrélativement l'augmentation du courant créé initialement dans le circuit. Ces dispositifs ainsi conçus ne permettent pas de récupérer avec un bon rendement l'énorme énergie spécifique de l'explosif $(5 \mathrm{~kJ} / \mathrm{g})$.
Nous nous contenterons dans ce qui suit d'un rendement de conversion de l'explosif relativement faible (quelques pour cent) mais nos efforts porteront sur l'amélioration du rendement électrique proprement dit du circuit. Ce rendement dépend essentiellement de la perte de flux magnétique, des dimensions géométriques et du temps de fonctionnement. Les résultats présentés montrent la façon de définir un générateur de courant à rendement le plus élevé possible. Nous nous limitons aux générateurs solénoïdaux alimentant une charge inductive.

II. Principe des générateurs solénoïdaux. - Les générateurs de ce type se composent d'un solénoïde à pas variable et d'un tube coaxial central destiné au retour du courant. Ce tube contient un explosif puissant, amorcé à une extrémité. Sous l'action de l'explosif, le tube s'évase et vient fermer le circuit, l'autre

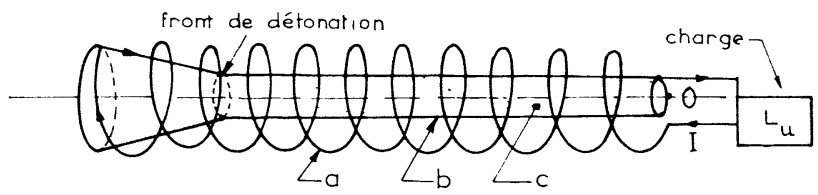

Frg. 1. - Principe du générateur à hélice et tube relevé : a) Solénoïde ; b) Tube relevé ; c) Explosif. 
extrémité étant fermée sur la charge. On réalise donc l'analogue d'un court-circuit mobile se déplaçant d'une extrémité à l'autre du solénoïde. La décharge d'une batterie de condensateurs établit le courant initial dans le circuit (voir Fig. 1).

III. Détermination des paramètres optimaux du solénoïde. - L'expérimentation, tout en donnant des résultats globaux sur les performances d'un montage, ne permet pas de déterminer le sens dans lequel doivent varier les paramètres géométriques (le pas en particulier) pour obtenir le courant le plus élevé et le meilleur rendement. Notre but est de calculer les valeurs du pas ainsi que la longueur du solénoïde en fonction des conditions imposées. Nous envisageons successivement deux cas : d'une part le solénoïde à pas variable continu, d'autre part, le solénoïde en étages, où le pas est constant dans chaque étage mais varie d'un étage au suivant [1]. La première réalisation, plus difficile sur le plan technologique a été entreprise par Sakharov [2], par Crawford et Damerov [3] par Shearer et al. [4] ainsi que dans notre laboratoire.

1. BASES DE CALCUL. - En première approximation on assimile le générateur à un circuit de résistance $R(z)$ et d'inductance $L(z)$ variables, parcourues par le même courant $I$. Nous ne tiendrons compte que des pertes par effet Joule; nous montrerons en effet, par la suite, qu'il existe des pertes de flux qui ne peuvent rentrer dans ce cadre, mais qui sont en général inférieures d'au moins un ordre de grandeur aux pertes par effet Joule.

Soit $L I$ le flux total du circuit :

$$
\frac{\mathrm{d}}{\mathrm{d} t}(L I)=-R I \text {. }
$$

L'origine de l'abscisse est choisie au point O, extrémité du solénoïde fermée sur la charge; on affecte l'indice 0 aux valeurs des variables en début de compression et l'indice $l$ à ces valeurs en fin de compression. L'origine des temps est l'instant où l'onde de choc passe en $\mathrm{O}$. Il vient :

$$
I=I_{0} \frac{L_{0}}{L} \exp \left(-\int_{t_{0}}^{t} \frac{R}{L} \mathrm{~d} t\right) .
$$

Il convient pour définir la variation du courant $I$ de calculer l'inductance et la résistance totale du circuit, en fonction de la densité de spires $n(z)$ et des paramètres électriques du circuit : $\sigma$ la conductivité du matériau, $\delta$ l'épaisseur de peau, $R_{e}, R_{i}$ et $S$ les

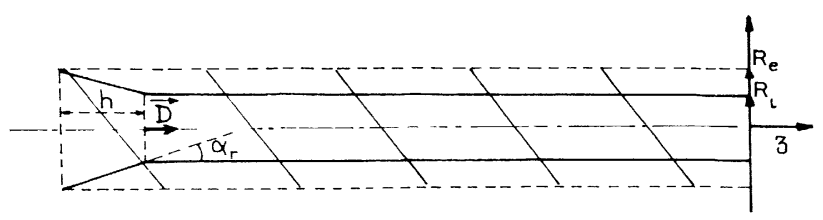

FIG. 2. - Notations du générateur à hélice. dimensions. $R_{l}$ et $L_{l}$ sont les valeurs à la limite de $R$ et $L$.

$$
\begin{gathered}
L(z)=\mu_{0} S \int_{z}^{0} n^{2}(z) \mathrm{d} z+L_{l} \\
R(z)=\frac{2 \pi\left(R_{e}+R_{i}\right)}{\sigma \delta} \int_{z}^{0} n^{2}(z) \mathrm{d} z+R_{l} .
\end{gathered}
$$

Ces formules ne sont valables que dans un certain domaine qui sera précisé plus loin.

La formule (4) tient compte des pertes par induction dans le tube, et suppose implicitement que les spires sont jointives.

2. CRITÈRE DE BON FONCTIONNEMENT. - Le champ magnétique dans la région du tube relevé, doit conserver une valeur moyenne ou du moins ne pas dépasser une valeur donnée, pour les raisons suivantes :

a) La diffusion du champ au travers du tube est un phénomène non linéaire en raison de l'échauffement et de l'augmentation de résistivité qui en résulte.

b) La pression magnétique $P_{\mathrm{M}}$, établie devant le tube relevé et proportionnelle au carré du champ, ne peut atteindre une valeur trop élevée sans risque de voir cette partie du tube rebondir ou du moins présenter des instabilités du type de celles de Taylor. En cas d'instabilités la formation de contacts multiples entre tube relevé et solénoïde a des chances de se produire et entraînerait alors l'existence de poches de flux perdu au détriment du rendement général. En d'autres termes la pression $P_{\mathrm{M}}$ ne doit pas dépasser la valeur $P_{e}$ de la pression des gaz d'explosif poussant le tube. Dans cette hypothèse la propagation du relèvement à la vitesse $D$ s'opère sous un angle $\alpha_{r}$ constant et le régime de fonctionnement est stationnaire. On écrit par suite que la puissance électromagnétique du circuit est au plus égale à la puissance dégagée par l'explosif :

$$
\frac{\mathrm{d}}{\mathrm{d} t} \frac{\left(L I^{2}\right)}{2}+R I^{2} \leqslant P_{e} S D .
$$

A l'aide de (1) on déduit :

$$
-I^{2} \frac{\mathrm{d} L}{\mathrm{~d} t} \leqslant 2 P_{e} S D
$$

Finalement en utilisant (4), on fait apparaître un critère dans lequel intervient le pas du solénoïde, ou son inverse $n(z)$, et le courant $I(z)$ soit :

$$
n I \leqslant n_{l} I_{l}=\sqrt{\frac{2 P_{e}}{\mu_{0}}} .
$$

3. SolÉnOIde A PAS VARIABle CONTINU. - D'après (6), les conditions de fonctionnement les meilleures seront obtenues si, quel que soit $\mathrm{z}$, l'égalité suivante est vérifiée :

$$
n I=n_{l} I_{l}
$$


En faisant intervenir cette condition dans l'équation du circuit :

$$
\frac{\mathrm{d}}{\mathrm{d} z}(L I)+\frac{R}{D} I=0
$$

on trouve l'expression générale :

$$
\begin{aligned}
& {\left[\mu_{0} S \cdot \frac{1}{n} \cdot \frac{\mathrm{d}}{\mathrm{d} z}\left(\begin{array}{l}
1 \\
n
\end{array}\right)+\frac{2 \pi\left(R_{e}+R_{i}\right)}{D \sigma \delta} \cdot \frac{1}{n^{2}}\right] \times} \\
& \quad \times \int_{z}^{0} n^{2}(z) \mathrm{d} z+\frac{R_{l}}{D} \cdot \frac{1}{n^{2}}+L_{l} \cdot \frac{1}{n} \frac{\mathrm{d}}{\mathrm{d} z}\left(\frac{1}{n}\right)-\mu_{0} S=0 .
\end{aligned}
$$

Il est commode d'introduire des variables géométriques sans dimension rapportées à la longueur caractéristique :

$$
Z_{0}=\frac{I_{l}^{2} L_{l}}{2 P_{e} S}=\frac{L_{l}}{\mu_{0} S n_{l}^{2}}
$$

$\zeta=\frac{z}{Z_{0}} \quad$ variable réduite

$v=\frac{n}{n_{l}}=\frac{I_{l}}{I} \quad$ gain en courant

$\Lambda=\frac{L}{L_{l}} \quad$ variable réduite

$$
k=\frac{D \mu_{0} S \sigma \delta}{Z_{0} 2 \pi\left(R_{e}+R_{i}\right)}=\frac{D}{Z_{0}} \frac{\mathrm{d} L}{\mathrm{~d} R}
$$

caractérise les pertes dans le circuit

$$
\rho=\frac{R_{l} \sigma \delta}{Z_{0} 2 \pi\left(R_{e}+R_{i}\right) n_{l}^{2}}=k \frac{R_{l}}{L} \cdot \frac{Z_{0}}{D}=\frac{R_{l}}{L_{l}} \frac{\mathrm{d} L}{\mathrm{~d} R}
$$
caractérise l'impédance de la charge

$u(z)=\frac{n_{l}^{2}}{n^{2}}=\frac{1}{v^{2}} \quad$ variable réduite

L'équation (7) devient :

$$
\left[\frac{1}{2} \frac{\mathrm{d} u}{\mathrm{~d} \zeta}+\frac{u}{k}\right] \int_{\zeta}^{0} \frac{\mathrm{d} \zeta}{u}+\frac{\rho}{k} u+\frac{1}{2} \frac{\mathrm{d} u}{\mathrm{~d} \zeta}-1=0 .
$$

Cette équation admet pour solution générale :

$$
u=\frac{\beta}{\alpha^{2}} \operatorname{sh}^{2} \frac{\alpha}{\sqrt{2}}\left(\zeta+\zeta_{0}\right)
$$

Les valeurs des constantes $\alpha, \beta, \zeta_{0}$ sont obtenues en calculant les expressions :

$$
\begin{aligned}
\frac{\mathrm{d} u}{\mathrm{~d} \zeta} & =\frac{\beta}{\alpha} \sqrt{2} \operatorname{sh} \frac{\alpha}{\sqrt{2}}\left(\zeta+\zeta_{0}\right) \operatorname{ch} \frac{\alpha}{\sqrt{2}}\left(\zeta+\zeta_{0}\right) \\
\int_{\zeta}^{0} \frac{\mathrm{d} \zeta}{u} & =\frac{\alpha \sqrt{2}}{\beta}\left[\operatorname{coth} \frac{\alpha}{\sqrt{2}}\left(\zeta+\zeta_{0}\right)-\operatorname{coth} \frac{\alpha}{\sqrt{ } /} \zeta_{0}\right] .
\end{aligned}
$$

En reportant dans l'équation (9), on obtient une équation linéaire en th $\frac{\alpha}{\sqrt{2}}\left(\zeta+\zeta_{0}\right)$ qui est vérifiée identiquement si les deux conditions suivantes sont satisfaites :

$$
\frac{\beta}{\alpha^{2}}=k \frac{1-\frac{2}{\alpha^{2} k^{2}}}{1-\rho}
$$

$$
\operatorname{coth} \frac{\alpha}{\sqrt{2}} \zeta_{0}=\frac{\alpha}{\sqrt{2}} k \frac{1-\frac{2 \rho}{\alpha^{2} k^{2}}}{1-\rho} .
$$

D'autre part, les conditions finales imposent :

$$
\frac{\beta}{\alpha^{2}} \operatorname{sh}^{2} \frac{\alpha}{\sqrt{2}} \check{\zeta}_{0}=1 \text {. }
$$

Par identification entre (11) et (12), il vient :

$$
\left\{\begin{array}{l}
\alpha^{2}=\frac{2}{k}\left(\frac{\rho^{2}}{k}+1-\rho\right) \\
\frac{\beta}{\alpha^{2}}=\frac{k(k-\rho-1)}{\rho^{2}+k(1-\rho)} .
\end{array}\right.
$$

On en déduit facilement

$$
v=\frac{\operatorname{sh} \frac{\alpha}{\sqrt{2}} \zeta_{0}}{\operatorname{sh} \frac{\alpha}{\sqrt{2}}\left(\zeta+\zeta_{0}\right)}
$$

avec

$$
\frac{\alpha}{\sqrt{2}} \zeta_{0}=\left[\operatorname{Argsh} \frac{k(k-\rho-1)}{\rho^{2}+k(1+\rho)}\right]^{-1 / 2} .
$$

On définit le gain en énergie $g$ comme le quotient de l'énergie récupérable $\frac{1}{2} L_{l} I_{l}^{2}$ par la somme des énergies fournies initialement au générateur, soit : $\frac{1}{2} I_{0} I_{0}^{2}+P_{e} S l$. L'expression générale donnant le gain $g$ est la suivante :

$$
\begin{aligned}
\frac{1}{g}=\frac{1}{2} \frac{k}{\rho^{2}+} & k(1-\rho) \\
& +\frac{l}{Z_{0}}+ \\
& \frac{2 \rho^{2}+k-2 k \rho}{2\left[\rho^{2}+k(1-\rho)\right]} \operatorname{ch}\left[\alpha \sqrt{2} \frac{l}{Z_{0}}\right] \\
& -\frac{k}{2 \sqrt{\rho^{2}+k(1-\rho)}} \operatorname{sh}\left[\alpha \sqrt{2} \frac{l}{Z_{0}}\right] .
\end{aligned}
$$

Le gain $g$, pour des valeurs données de $k$ et $\rho$, dépend essentiellement du rapport $l / Z_{0}$.

L'équation (14) se simplifie dans le cas où $\rho=1$. En effet le rapport $R / L$ est indépendant du temps d'après (8), et il vient : $R / L=R_{l} / L_{l}$; le gain $v$ s'écrit alors :

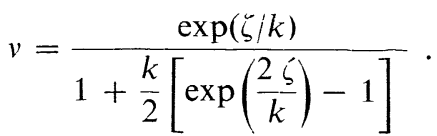


Le gain $g$ prend la forme :

$$
\frac{1}{g}=\left(1-\frac{k}{2}\right) \operatorname{ch}\left(\frac{2}{k} \frac{l}{Z_{0}}\right)+\frac{k}{2}\left[1-\operatorname{sh}\left(\frac{2}{k} \frac{l}{Z_{0}}\right)\right]+\frac{l}{Z_{0}}
$$

Les variations du gain $v$ pour différentes valeurs de $\rho$ et $k$ apparaissent sur le diagramme en coordonnées semi-logarithmiques de la figure 3 . On remarque que le coefficient d'impédance $\rho$ ne joue un rôle important que dans le choix du pas près de la charge $L_{u}$; par contre $\rho$ a peu d'importance près du point d'amorçage ; cette propriété apparaît sur la figure 3 en observant que pour une même valeur de $k$, les courbes sont quasisuperposables dans la région proche de leur asymptote.

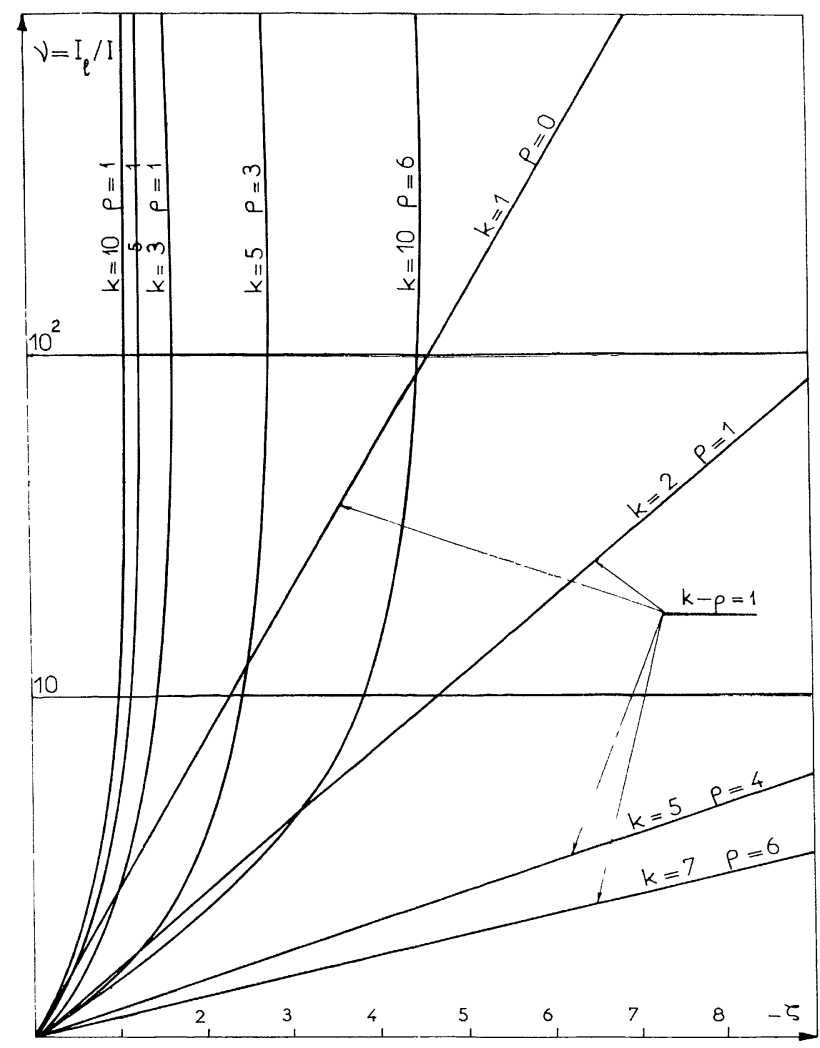

FIG. 3. - Variation optimale de la densité de spires pour un solénoïde à pas variable; la fonction 14 est représentée pour différentes valeurs de $k$ et de $\rho$.

Dans le cas où $k-\rho=1$, l'équation (14) prend la forme simple suivante $: v=\exp (\zeta / k)$. Cette expression donne sur le diagramme de la figure 3, des droites; ce cas correspond à une densité de spires variant exponentiellement avec l'abscisse ; en effet la variable $u$ s'exprime ici par la formule : $u=\exp \left(\frac{2}{k} \zeta\right)$. Le gain $g$ est donné par la nouvelle expression :

$$
\frac{1}{g}=\left(1-\frac{k}{2}\right) \exp \left(-\frac{2}{k}-\frac{l}{Z_{0}}\right)+\frac{k}{2}+\frac{l}{Z_{0}} .
$$

Les calculs effectués jusqu'à présent utilisent les expressions (3) et (4) qui ne sont valables que sous deux conditions :

a) le champ magnétique le long du solénoïde n'a qu'une composante axiale ce qui entraîne :

$$
n(z)>\frac{1}{R_{i}}
$$

b) en une section droite du solénoïde, le champ est homogène ce qui s'exprime par :

$$
\left|\frac{\partial n}{\partial z}\right|<\frac{1}{R_{e}^{2}} .
$$

En fait ces conditions sont en défaut en deux régions du solénoïde :

- vers la charge, car la condition (16) s'écrit :

$$
n(z) \approx \frac{1}{R_{e}},
$$

- vers le point d'amorçage car la condition (17) devient :

$$
\left|\frac{\partial n}{\partial z}\right| \approx \frac{1}{R_{l}^{2}}
$$

Près de la charge, on peut conserver la méthode générale, sous réserve de substituer à $n$ une densité fictive de spires $n^{\prime}$ donnée par la formule :

$$
n^{\prime}(z)=\left[n^{2}(z)+\frac{1}{2 \pi S} \log \frac{R_{e}}{R_{i}}\right]^{1 / 2} .
$$

Près du point d'amorçage, la condition (17) indique que la variation du pas doit être faible. En pratique cette variation n'est pas très élevée ; il est en effet délicat de construire un solénoïde à spires très serrées et de plus l'espace entre spires doit rester supérieur à une certaine limite pour éviter tout risque de claquage électrique.

Dans la région centrale du solénoïde, les conditions (16) et (17) sont bien remplies et les courbes de gain $v$ restent valables; elles indiquent pour une valeur de $v$ fixée, la façon dont la longueur $l$, exprimée par la variable $\zeta$, dépend du choix des coefficients $k$ et $\rho$.

4. SolÉnOIdes GIGOGNes - Pour des raisons technologiques il est plus facile de réaliser un solénoïde constitué de plusieurs étages gigognes présentant chacun leur propre pas constant. Dans cette nouvelle hypothèse, le critère (6) est satisfait au mieux si $n I=n_{l} I_{l}$ en fin de chaque étage.

Le problème d'optimisation du générateur se présente de la façon suivante : étant donnés $N$ étages à pas constant et un gain total en courant $I / I_{N}$ fixé, il s'agit de déterminer la longueur et le pas de chaque étage afin d'obtenir la longueur totale la plus faible possible.

Les tronçons sont numérotés comme l'indique la figure 4 , soit $L\left(z_{i}\right)=L_{i}$, l'impédance des tronçons jusqu'à la charge. La variation du courant pendant la 


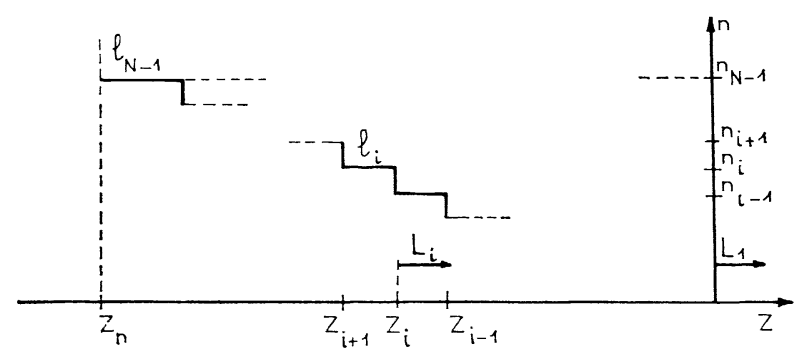

Fig. 4. - Représentation schématique d'un solénoïde gigogne.

compression de l'étage $i$ est donnée par l'équation (2). Pour ne pas alourdir les calculs, nous supposerons que $R / L$ est constant (cette condition impose : $\rho=1$ ).

Pour $z_{i+1}<z<z_{i}$ on obtient :

$$
I=I_{i+1} \frac{L_{i+1}}{L} \exp \left[-\frac{R}{L D}\left(z-z_{i+1}\right)\right]
$$

d'où

$$
\frac{I_{i}}{I_{i+1}}=\frac{L_{i+1}}{L_{i}} \exp \left[-\frac{R}{L D} l_{i}\right]
$$

D’autre part :

$$
L_{i+1}=L_{i}+\mu_{0} S n_{i}^{2} l_{i}
$$

Le critère (6) impose :

$$
\frac{I_{i}}{I_{i+1}}=\frac{n_{i+1}}{n_{i}}
$$

En notant $\lambda_{i}=l_{i} / Z_{0}$ ces relations s'écrivent avec les variables réduites $(8)$ :

$$
\left\{\begin{array}{l}
\frac{v_{i+1}}{v_{i}}=\frac{\Lambda_{i+1}}{\Lambda_{i}} \exp \left(-\frac{\lambda_{i}}{k}\right) \\
\Lambda_{i+1}=\Lambda_{i}+v_{i}^{2} \lambda_{i} .
\end{array}\right.
$$

Tous les tronçons sont supposés optimisés, sauf ceux d'indices $i-1$ et $i$. Il faut déterminer $\lambda_{i-1}, \lambda_{i}$ et $v_{i}$ de façon à obtenir $\lambda_{i-1}+\lambda_{i}$ minimum, soit :

$$
\mathrm{d} \lambda_{i-1}+\mathrm{d} \lambda_{i}=0 \text {. }
$$

$v_{i+1}, v_{i-1}, \Lambda_{i-1}$ et $\Lambda_{i+1}$ sont connus et indépendants de $\lambda_{i}$ mais $\Lambda_{i}$ dépend de $\lambda_{i}$. Eliminons $\Lambda_{i}$ et $v_{i}$ dans les relations (21) écrites à l'ordre $i$ et $i+1$

$$
\begin{aligned}
\Lambda_{i+1}=\Lambda_{i-1}+\lambda_{i-1} v_{i-1}^{2}+\lambda_{i-1} v_{i-1}^{2}+\lambda_{i} v_{i-1}^{2} \times \\
\times\left[\frac{\Lambda_{i-1}+\lambda_{i-1} v_{i-1}^{2}}{\Lambda_{i-1}} \exp \left(\frac{\lambda_{i-1}}{k}\right)\right]^{2} .
\end{aligned}
$$

On obtient par différenciation :

$$
\lambda_{i}=\frac{1}{2} \frac{\exp \left(2 \frac{\lambda_{i-1}}{k}\right)-\left(\frac{\Lambda_{i}}{\Lambda_{i-1}}\right)^{2}}{\left(\frac{\Lambda_{i}}{\Lambda_{i-1}}\right)^{2}\left(\frac{1}{k}-\frac{v_{i-1}^{2}}{\Lambda_{i}}\right)} .
$$

En regroupant avec les relations (21) on obtient REVUE DE PHYSIQUE APPLIQUÉE. - T. 5, No 2, AVRIL 1970 des relations de récurrence qui permettent de calculer les étages en partant de la charge

$$
\begin{aligned}
& \Lambda_{i}=\Lambda_{i-1}+\lambda_{i-1} v_{i-1}^{2} \\
& v_{i}=v_{i-1} \frac{\Lambda_{i}}{\Lambda_{i-1}} \exp \left(-\frac{\lambda_{i-1}}{k}\right) \\
& \dot{\lambda}_{i}=\frac{\lambda_{i-1}}{2} \frac{1-\left(\frac{v_{i-1}}{v_{i}}\right)^{2}}{1-\frac{\lambda_{i-1}}{k}-\frac{\Lambda_{i-1}}{\Lambda_{i}}} .
\end{aligned}
$$

Les premiers termes sont $\Lambda_{0}=1, v_{0}=1, \lambda_{0}$ arbitraire. Remarquons que $v_{i}$ représente, en variables réduites, le pas de l'étage d'indice $i$, mais aussi le gain en courant des $i$ premiers étages (d'indice 0 à $i$ - 1). Pour exploiter commodément le système (23), nous avons tracé des abaques donnant $v$ pour différentes valeurs de $k$ en fonction de $-\zeta$.

On lit en abscisse la longueur du solénoïde entre la charge et le $n$-ième étage inclus et en ordonnée le pas des étages correspondants. La longueur $\lambda_{0}$ du premier étage étant arbitraire, on obtient deux faisceaux de courbes, l'un à nombre d'étages donné, l'autre à $\lambda_{0}$ donné. $k$ étant fixé, l'abaque permet de déterminer le pas et la longueur de chaque étage, lorsqu'on se donne le gain en courant et le nombre d'étages.

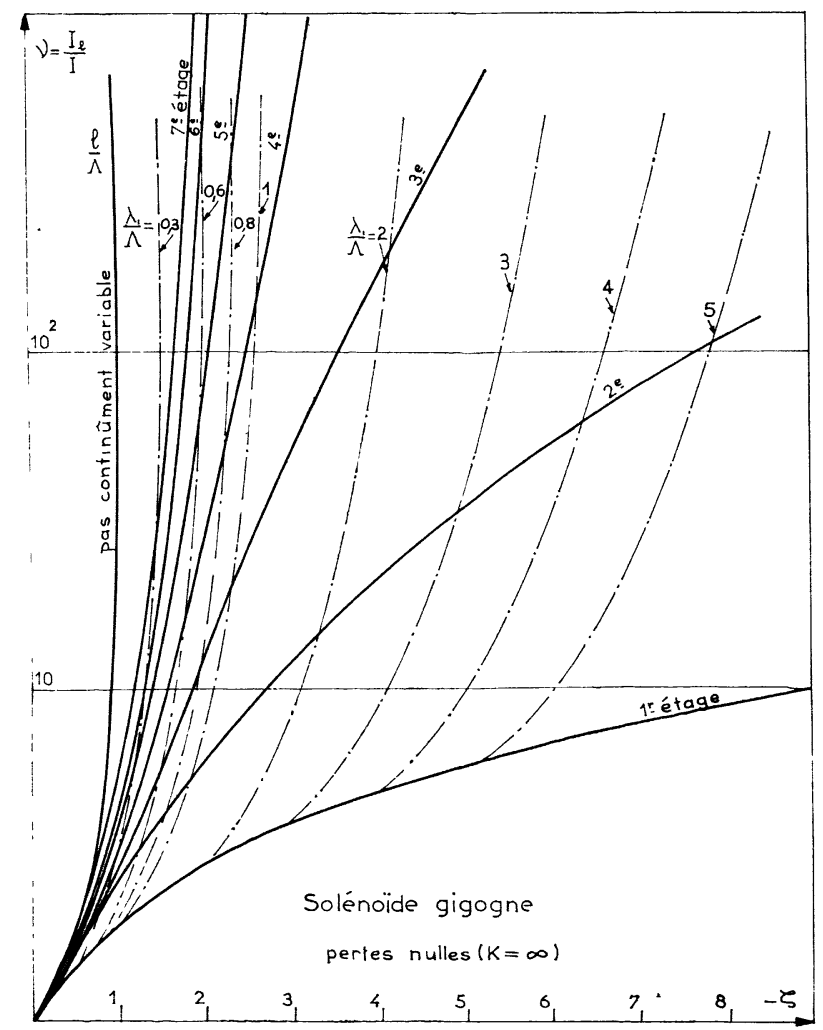

FIG. 5. - Abaque d'optimisation relatif à un solénoïde gigogne sans tenir compte des pertes. 


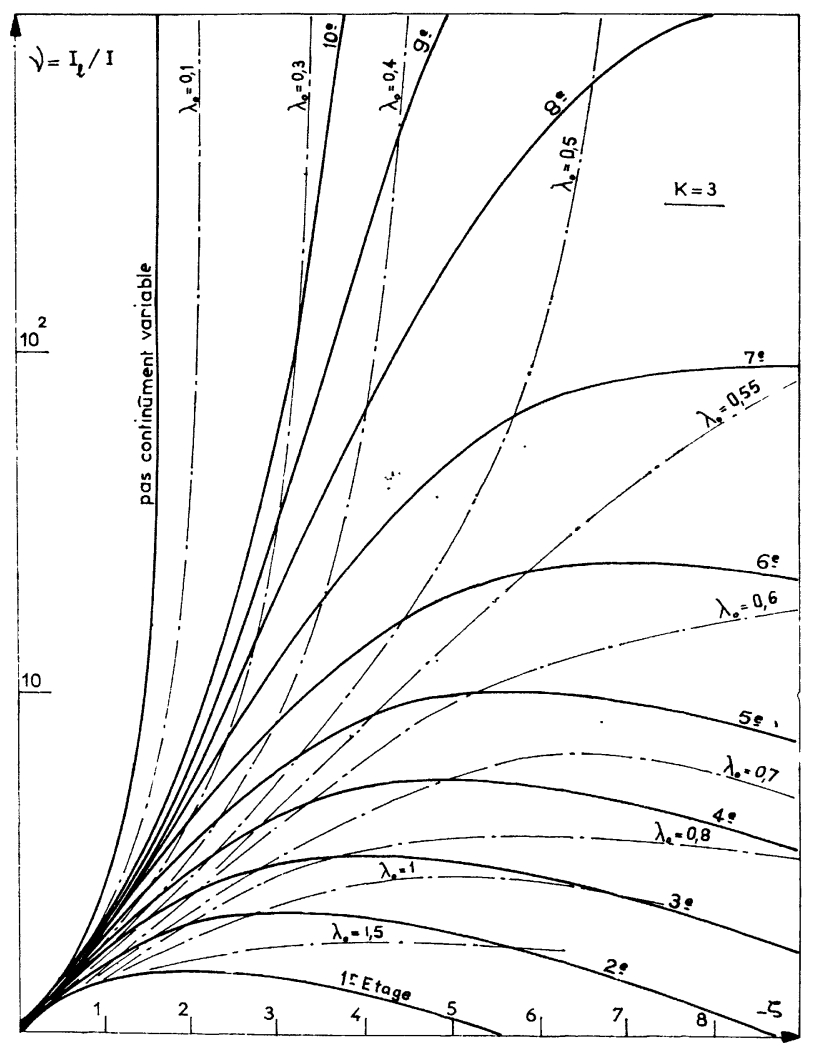

FIG. 6.

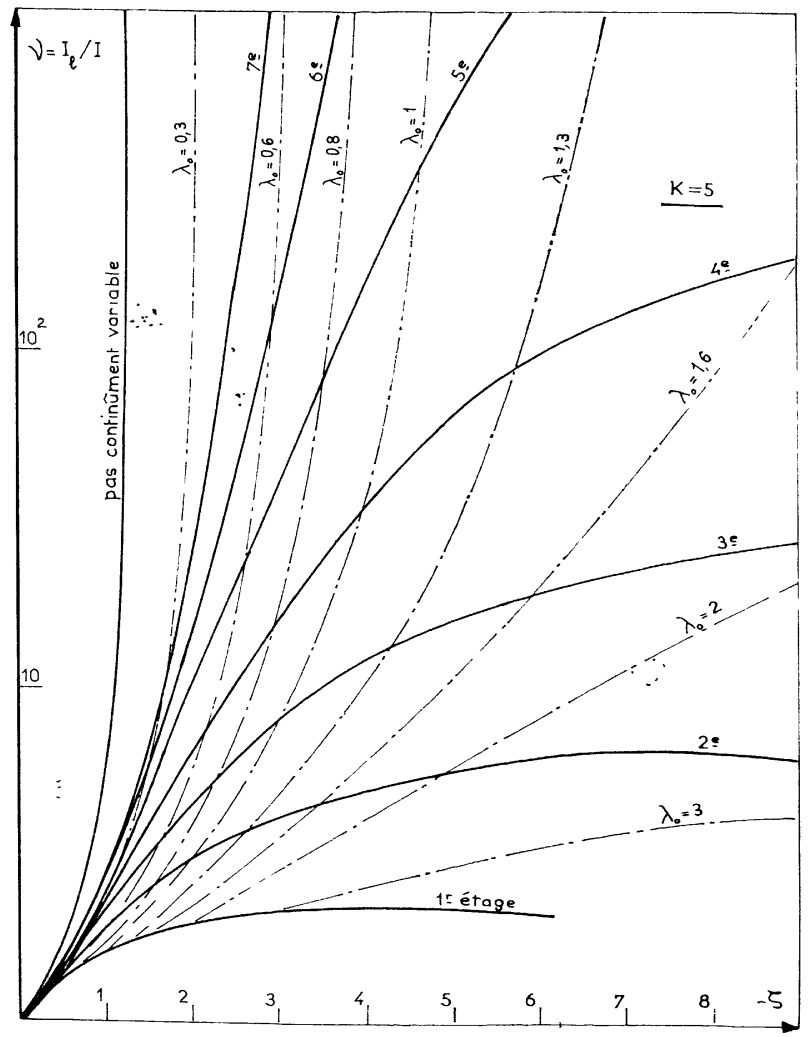

FIG. 7.
Par exemple, pour $k=5$, un gain de 90 et 5 étages :

$$
\begin{array}{ll}
v_{4}=16,5 & l_{4}=3,6-3=0,6 \\
v_{3}=5,7 & l_{3}=3-2,35=0,65 \\
v_{2}=2,7 & l_{2}=2,35-1,6=0,75 \\
v_{1}=1,55 & l_{1}=1,6-0,8=0,80 \\
v_{0}=1 & l_{0}=0,80 \quad=0,80 .
\end{array}
$$

L'abaque de la figure 5 représente la variation de $v$ en fonction de $-\zeta$ dans le cas où les pertes sont nulles $(k=\infty)$. On constate, dans ce cas idéal, que la longueur du solénoïde gigogne est nettement supérieure à celle $\mathrm{du}$ solénoïde à pas variable continu, mais l'allongement reste acceptable jusqu'à 4 ou même 3 étages. Pour $k=10$ (Fig. 6), l'allongement dû aux pertes est minime. En revanche, le réseau se déforme considérablement pour $k=5$ ou 3 (Fig. 7 et 8). Pour $k=2$, le problème n'a plus de solution intéressante. D'une façon générale, l'influence des pertes est beaucoup plus sensible pour les solénoïdes gigognes que pour le solénoïde optimisé à pas continûment variable.

IV. Pertes diverses. - Les pertes par effet Joule constituent la principale cause de diminution du flux dans le circuit. Il existe cependant de nombreuses autres

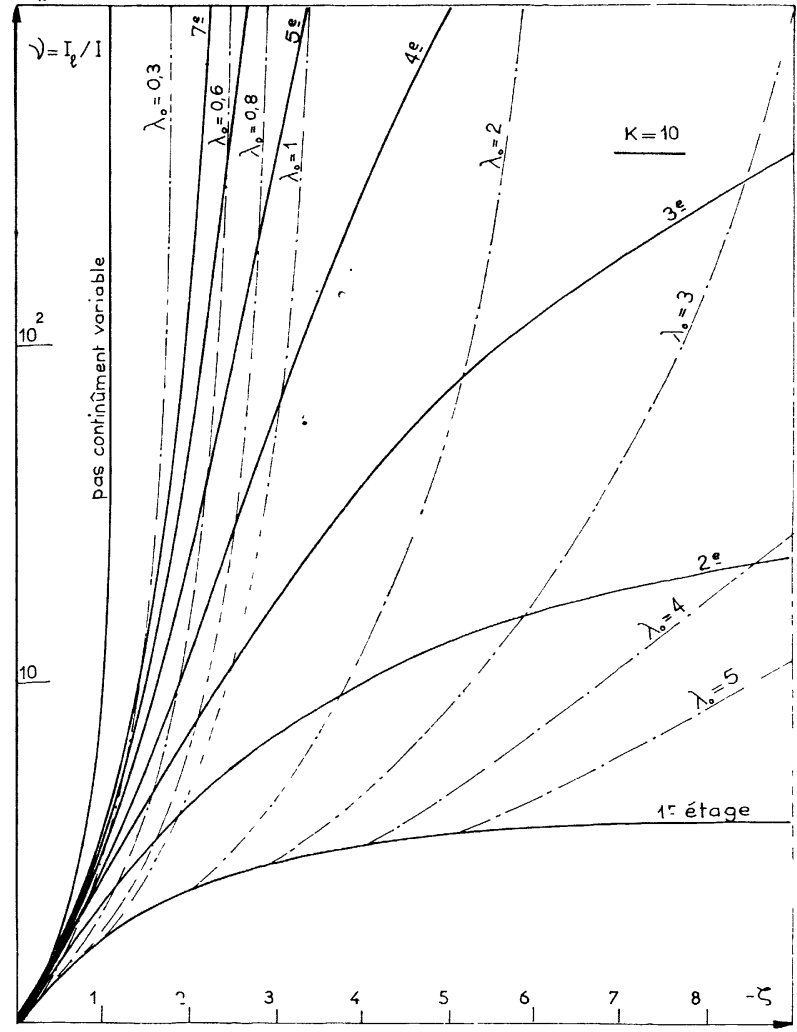

Fig. 6, 7, 8. - Abaques d'optimisation relatifs à un solénoïde gigogne où les pertes sont prises en compte. Les coordonnées sont sans dimension. La longueur de l'étage est représentée en abscisse; $v$ et le gain en courant sont proportionnels à la densité de spires du solénoïde. $\lambda_{0}$ est le paramètre attaché à chaque courbe et il représente la longueur du premier étage. Les pertes sont proportionnelles à $1 / k$. 
causes de pertes, non négligeables, dont Shearer [4] a donné une liste assez complète : compression des parois due à la pression magnétique, compression du gaz de remplissage, claquage diélectrique dans ce gaz. On peut faire une évaluation de ces différentes pertes par le calcul, mais les performances des générateurs réalisés sont inférieures aux prévisions théoriques.

On a alors envisagé d'autres causes possibles, telles que la présence d'un champ magnétique intense entre spires et la fissuration du tube relevé. En effet, lors de l'expansion du tube, des fissures peuvent se produire qui empêchent la circulation des courants induits et facilitent par conséquent la diffusion du champ vers l'intérieur du tube. D. B. Cummings [1] a montré expérimentalement que l'on peut atteindre un rapport d'expansion de 3 sur le diamètre du tube sans que des fissures ne s'ouvrent complètement si l'on choisit un matériau assez ductile. Les essais ont été effectués cependant sans champ magnétique; dans une expérience avec champ, la pression magnétique s'oppose à la progression de la surface libre du relèvement; cette condition est favorable au développement d'instabilités du type de Taylor, donc à la fissuration. Enfin, il se produit un glissement du relèvement sur luimême sous l'action des forces électromagnétiques. Nous allons calculer la force correspondante. En un point quelconque du tube relevé, nous introduisons deux composantes de la densité superficielle de courant (Fig. 9) :

$-J_{\varphi} \simeq n I \cos \alpha_{r}:$ composante azimutale due aux courants induits, tangente au cercle passant par $M$;

$-J_{\mathrm{m}}=I / 2 \pi r:$ composante méridienne, due au courant de retour.

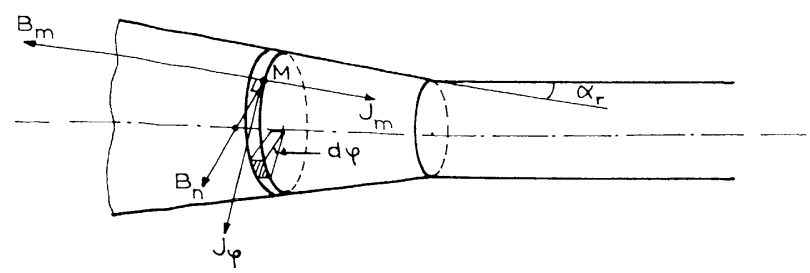

Fig. 9. - Composantes de la densité du courant superficiel et de l'induction magnétique.

De même l'induction magnétique présente deux composantes :

- $B_{\mathrm{m}}$ : tangent à la méridienne, discontinu au passage de la surface conductrice ;

$-B_{\mathrm{n}}$ : normal au relèvement et sans discontinuité.

L'action de $B_{\mathrm{m}}$ sur les deux composantes de la densité de courant correspond à la pression magnétique, contre laquelle travaille l'explosif. L'action de $B_{\mathrm{n}}$ sur $J_{\mathrm{m}}$ produit une force qui tend à faire tourner le relèvement. De $B_{\mathrm{n}}$ et $J_{\varphi}$ il résulte une force qui tend à faire glisser le relèvement sur lui-même dans la direction de $J_{\mathrm{m}}$.

Pour évaluer la perte correspondante, nous calculerons l'énergie cinétique communiquée au tube relevé, mais on aboutit également au résultat en passant par l'intermédiaire de la f. c. e. m. équivalente. Cette deuxième méthode, bien que plus délicate, montre mieux le mécanisme par lequel l'énergie est empruntée au circuit électrique.

Au point $M$, l'élément du relèvement $(\mathrm{d} z, \mathrm{~d} \varphi)$ est soumis aux forces infinitésimales suivantes :

$$
\begin{aligned}
& \mathrm{d}^{2} f_{\varphi}=\frac{I}{2 \pi} B_{\mathrm{n}} \frac{\mathrm{d} z}{\cos \alpha_{r}} \mathrm{~d} \varphi \\
& \mathrm{d}^{2} f_{\mathrm{m}}=r J_{\varphi} B_{\mathrm{n}} \frac{\mathrm{d} z}{\cos \alpha_{r}} \mathrm{~d} \varphi .
\end{aligned}
$$

La masse élémentaire correspondante vaut : $\mu \mathrm{d} \varphi \mathrm{d} z$ avec $\mu=\varpi e_{0} R_{i}$, où $\varpi$ est la masse spécifique et $e_{0}$ l'épaisseur du tube.

L'angle de relèvement étant faible, on admettra en première approximation que l'abscisse d'un point matériel reste constante.

Il en résulte les composantes de la vitesse :

$$
\begin{aligned}
& v_{\varphi}=\int_{z / D}^{t} \frac{I B_{n}}{2 \pi \mu \cos \alpha_{r}} \mathrm{~d} t \\
& v_{\mathrm{m}}=\int_{z / D}^{t} \frac{r J_{\varphi} B_{\mathrm{n}}}{\mu \cos \alpha_{r}} \mathrm{~d} t .
\end{aligned}
$$

L'énergie cinétique totale du relèvement s'en déduit :

$$
W_{\mathrm{c}}(t)=\int_{D t-h}^{D t} \pi \mu\left(v_{\varphi}^{2}+v_{\mathrm{m}}^{2}\right) \mathrm{d} z
$$

La puissance correspondante s'écrit :

$$
\begin{aligned}
\Pi_{\mathrm{c}}(t)=-D & \pi \mu\left[v_{\varphi}^{2}(D t-h)+v_{\mathrm{m}}^{2}(D t-h)\right]+ \\
+ & \int_{D t-h}^{D t} 2 \pi \mu\left(v_{\varphi} \frac{\partial v_{\varphi}}{\partial t}+v_{\mathrm{m}} \frac{\partial v_{\mathrm{m}}}{\partial t}\right) \mathrm{d} z .
\end{aligned}
$$

Le premier terme correspond à l'énergie perdue lorsque le relèvement atteint le solénoïde, le deuxième terme correspond à l'énergie $P_{\mathrm{c}}$ empruntée effectivement au circuit. En général $v_{\varphi}$ est petit devant $v_{\mathrm{m}}$ car $r J_{\varphi} / I \simeq r n \gg 1$ sauf éventuellement pour les spires les plus proches de la charge.

Comme nous ne cherchons qu'une évaluation approchée du terme de perte dans l'équation du circuit, nous négligerons les termes en $v_{\varphi}$.

Le calcul de ce terme de perte est particulièrement facile lorsque le solénoïde est optimisé avec un pas continu. Dans ce cas, le régime est pratiquement stationnaire : $n I, J_{\varphi}, B$, ne dépendent que de $X=D t-z$ et de $r$, considérés comme variables indépendantes. D'autre part, le relèvement est représenté par :

$$
r=R_{i}+X \operatorname{tg} \alpha_{r}
$$

(en supposant $\alpha_{r}$ constant). D'où

$$
v_{\mathrm{m}}=\int_{0}^{X} \frac{r(X) J_{\varphi}(X) B_{\mathrm{n}}(X)}{D \mu \cos \alpha_{r}} \mathrm{~d} X .
$$

Il en résulte que $W_{\mathrm{c}}$ ne dépend pas du temps et que $\Pi_{\mathrm{c}}$ est nul. 
La puissance perdue par le circuit vaut donc :

$$
P_{\mathrm{c}}=\pi D \mu\left[\int_{0}^{h} \frac{r J_{\varphi} B_{\mathrm{n}}}{D \mu \cos \alpha_{r}} \mathrm{~d} X\right]^{2}
$$

or

$$
B_{\mathrm{n}}=\frac{\cos \alpha_{r}}{2 \pi r} \frac{\partial \Phi_{i}}{\partial z}
$$

$\Phi_{i}$ représentant le flux au travers du cercle d'axe $\mathrm{O}_{z}$ passant par $M$. D'après l'hypothèse du régime stationnaire :

$$
\frac{\partial \Phi_{i}}{\partial z}=-\frac{1}{D} \frac{\partial \Phi_{i}}{\partial t}=\frac{J_{\varphi}}{D} \frac{2 \pi r}{\sigma \delta}
$$

d'où :

$$
P_{\mathrm{c}}=\frac{1}{4 \pi} \cdot \frac{P_{J}^{2}}{\mu D^{3}} \text { avec } P_{J}=2 \pi \int_{0}^{h} \frac{J_{\varphi}^{2} r}{\sigma \delta} \mathrm{d} X .
$$

$P_{J}$ représente la puissance Joule perdue dans le relèvement. On peut prendre l'expression approchée :

$$
P_{J}=\frac{(n I)^{2} 2 \pi\left(R_{0}^{3}-R_{i}^{3}\right)}{3 e_{0} \sigma R_{i} \operatorname{tg} \alpha_{r}} .
$$

Une évaluation de $P_{J}$ plus précise ferait intervenir les autres causes de pertes qui ont été évoquées et qui se traduisent par une augmentation de résistivité et une variation de l'épaisseur de peau.

Pour $R_{e}=5 \mathrm{~cm}, R_{i}=3 \mathrm{~cm}, e_{0}=2 \mathrm{~mm}$,

$$
n I=5 \times 10^{7} \mathrm{~A} / \mathrm{m} \text {, }
$$

on évalue : $P_{J}=6 \times 10^{9} \mathrm{~W}$, soit le $1 / 10^{\mathrm{e}}$ de la puissance récupérable de l'explosif $\left(P_{e} S D\right)$.

Dans ces conditions, $P_{\mathrm{c}}\left(10^{7} \mathrm{~W}\right)$ est négligeable par rapport à la perte ohmique, mais semble d'une importance comparable à la perte par compression des parois. Si la diffusion du champ magnétique est accrue par des effets tels que des amorces d'instabilités ou de fissuration, le terme de perte $P_{c}$ augmente considérablement puisqu'il varie comme le carré de la perte Joule. Cette perte peut donc être légitimement négligée dans les calculs d'optimisation, mais il faut en tenir compte pour expliquer le fonctionnement des générateurs de hautes performances.

V. Conclusion. - Malgré les approximations que nous avons dû consentir, principalement sur le calcul des pertes, la méthode d'optimisation du pas peut apporter un gain substantiel dans la réalisation de générateurs de courant puissants, surtout lorsque des expériences correspondantes permettent d'ajuster la valeur numérique des paramètres.

\section{Bibliographie}

[1] Cummings (D. B.), Morley (M. J.), Electrical pulses from helical and coaxial explosive generators. Proceedings of the Conference on Megagauss Magnetic Field Generation by Explosives and Related Experients, Frascati, Italy, september, 21-23, 1965, EUR 2750, e, 451-470.

[2] Sakharov (A. D.), Magnetoimplosive Generators, Sov. Phys. Usp., 1966, 9, 2, 294-299.
[3] Crawford (J. C.), Damerov (R. A.), Explosively Driven High Energy Generators, J. A. P., 1968, 39, 11, 5224-5231.

[4] Shearer (J. W.) et alii, Explosive Driven Magnetic Field Compression Generators, J. A. P., 1968, 39, 4, 2102-2116. 